\title{
Research trends in biomimetic medical materials for tissue engineering: commentary
}

\author{
Ki Dong Park ${ }^{1 \dagger}$, Xiumei Wang ${ }^{2 \dagger}$, Jae Young Lee ${ }^{3}$, Kyung Min Park ${ }^{4}$, ShengMin Zhang ${ }^{5^{*}}$ and Insup Noh ${ }^{6,7^{*}}$
}

\begin{abstract}
We introduce our active experts' communications and reviews (Part II) of 2015 Korea-China Joint Symposium on Biomimetic Medical Materials in Republic of Korea, which reflect their perspectives on current research trends of biomimetic medical materials for tissue regeneration in both Korea and China. The communications covered three topics of biomimetics, i.e., 1) hydrogel for therapeutics and extracellular matrix environments, 2) design of electrical polymers for communications between electrical sources and biological systems and 3) design of biomaterials for nerve tissue engineering. The reviews in the Part II will cover biomimetics of 3D bioprinting materials, surface modifications, nano/micro-technology as well as clinical aspects of biomaterials for cartilage.
\end{abstract}

\section{Introduction}

An invitation-based, bilateral symposium on biomimetic medical materials was held from October 22 to 26 in Seoul, Korea, towards building strong relationships among established leaders and emerging young scientists in Korea and China. Numerous breakthrough achievements in biomedical materials and nano-biotechnology research on regenerative medicine have been made in the two countries throughout successful series of the previous Korea-China Joint Symposium on Biomaterials and Nano-biotechnology pioneered by professors Inseop Lee in Yonsei University of Korea and Fu-Zhai Cui in Tsinghua University of China over more than 10 years.

Biomaterials science and technology have recently evolved into a new stage of tissue engineering research through the modeling of characteristics of the extracellular matrix (ECM) of defect tissues as well as the advancement of new fabrication methods of 3D bioprintings of functional biomaterials. The symposium focused on recent scientific progress on biomimetics and regenerative medical materials for tissue engineering,

\footnotetext{
* Correspondence: smzhang@hust.edu.cn; insup@seoultech.ac.kr

${ }^{\dagger}$ Equal contributors

${ }^{5}$ Advanced Biomaterials and Tissue Engineering Center, Huazhong University of Science and Technology, Wuhan, P. R. China

${ }^{6}$ Convergence Institute of Biomedical Engineering and Biomaterials, Seoul

National University of Science and Technology, Seoul 01811, Korea

Full list of author information is available at the end of the article
}

particularly bone, cartilage and nerve, and provided a platform for the academic cooperation between Korea and China.

During the discussion session about two countries' scientific collaboration led by key scientists, diverse issues were mainly discussed on how to upgrade our scientific relationship of this new research area. Other topics also raised how to contribute to improve the relationships among the National Research Foundation (NRF) of Korea and the National Natural Science Foundation of China (NSFC), Korean Society for Biomaterials (KSBM) and Chinese Society for Biomaterials (CSBM) and how to improve partnerships in research and industrial aspects.

The key members of both Korean and Chinese Societies for Biomaterials agreed on holding a biannual exchange forum of young scientists during each society's annual meeting. The first forum was held on November 20, 2015 in Hainan, China, and the second one is scheduled to be held in Korea in 2016.

Herein, we introduce our active experts' communications and reviews, which reflect their perspectives on current research trends of biomimetic medical materials for tissue regeneration. The communications covered three topics of biomimetics, i.e., 1) hydrogel for therapeutics and extracellular matrix environments, 2) design of electrical polymers for communications between 
electrical sources and biological systems and 3) design of biomaterials for nerve tissue engineering. The reviews will cover biomimetics of 3D bioprinting materials, surface modifications, nano/micro-technology as well as clinical aspects of biomaterials for cartilage.

\section{Biomimetic medical materials trends: polymeric hydrogels}

Polymeric hydrogels have received substantial attentions in various biomedical applications due to their structural similarities to the native extracellular matrix (ECM) as well as multi-tunable properties. Up to date, various kinds of polymeric hydrogels have been developed through physical- or chemical-cross linking mechanisms, which can be applied as either therapeutic implants or therapeutic vehicles for controlled drug delivery and tissue regeneration [1]. Specifically, the engineered polymeric hydrogels have been widely used either as therapeutic delivery carriers that facilitate tissue regeneration and repair or as an artificial extracellular microenvironment that support 3D cells or organs growth [2,3]. Here, we briefly discuss the emerging trends in polymeric hydrogel materials for biomedical research fields.

\section{Therapeutic vehicles}

In the past decade, polymeric hydrogels have been used as a carrier to deliver therapeutic agents (e.g., growth factors, cells or other bioactive molecules) for tissue regeneration [4]. Recently, researchers have focused on developing advanced hydrogel materials that allow much finer control over the spatial and temporal delivery of the therapeutic agents to improve the therapeutic efficacy. These engineered hydrogel materials can play a role not only as delivery vesicles for the therapeutic agents, but also to direct stimulate tissue regeneration and repair through physicochemical interactions between the materials and the host tissues. Park and his colleagues have developed a micro/nanogel composed of in situ crosslinkable gelatin-based microgel and selfassembled heparin-based nanogels, which can serve as an injectable growth factor delivery carrier for the urethral muscle regeneration [5]. Interestingly, they noticed that incorporating a growth factor -encapsulated heparinnanogels into a gelatin matrix allowed the hydrogel composites to release the therapeutic agent continuously up to 4 weeks, resulting in enhanced urethral muscle regeneration and recovery of their biological function. More recently, Park and Gerecht have developed hypoxiainducible hydrogels that can provide artificial hypoxic microenvironment when injected into the body, showing facilitating vascular tissue regeneration and tissue augmentation [6]. These innovative approaches have considerable values, and therefore hold great potential for tissue regenerative medicine.

\section{Artificial extracellular microenvironments}

Recently, many researchers are interested in utilizing the artificial extracellular microenvironment using biomimetic hydrogel materials to support cell growth. These engineered cellular microenvironments are created by tailoring the polymeric backbone with cellular response molecules (e.g., cell adhesion peptides or proteolytic-cleavable peptides), which are critical for supporting 3D cell growth [7]. In addition, it is well known that the native cellular microenvironment contains spatial-gradients in various physicochemical properties, including matrix proteins, oxygen gradients, mechanical strength, and microstructure properties $[8,9]$. Combining these parameters, the synthetic microenvironments have been utilized either to create three dimensional tissue constructs for tissue regeneration or to generate engineered disease models (e.g., engineered tumor, vascular, skin, and liver models) for a better understanding of basic cellular/molecular biology and clinical outcomes. More recently, the engineered tissue constructs have been designed by a combination of polymeric hydrogels with micro-/nano-fabrication techniques (e.g., microfluidics and 3D printing) to more accurately recapitulate the complexity of the native cellular environments [10-12].

\section{Biomimetic medical materials trends: design of electrical polymers for biomimetics}

Tissues and cells in our body are strongly affected by electrical signals, such as electrical field and current, and at the same time utilize electrical signals as important factors that control physiological conditions [13]. For example, embryonic body development and tissue regeneration are known to involve electrical signals [14]. The simple examples include electrically excitable cells, such as neurons, cardiomyocytes, myoblasts, utilize electrical signals for the inherent functions [15]. Surprisingly, recent studies have revealed that other types of cells, such as stem cells, also respond to electrical stimulation and exhibit various cell behaviors [16]. Accordingly, there have been growing attentions to an effective way to deliver or record electrical signals to or from biological systems. To effectively mediate electrical signals between electrical sources (e.g., electrodes) and biological systems, materials that possess good electrical conductivity are required. Various conductive materials, including metals, metal oxides, carbon nanomaterials, and conductive polymers, have been extensively utilized to construct conductive interfaces. Among them, electrically conductive materials, polypyrrole (PPy), polythiophene (PT), and PEDOT, are conductive organic materials [17]. Compared to other conductive materials, these conductive polymers offer some important advantages, such as biocompatibility, ease in modification, lower rigidity, and redox activity. Consequently, these conductive polymers 
have been widely used as a whole materials or components in tissue scaffolds, sensors, biolectrodes, and so forth.

Native tissue and its environments are highly organized and their properties are harmonized to permit cues modulating biological activities. Depending on the physiological and pathological states, native environments actively respond and dynamically change their composition, structures, and bioactivities [18]. Therefore, profound effects have been made to incorporate the properties of the native environments such as ECM into biomaterials to create 'artificial' environments, of which strategy is known as 'biomimetics'. Likewise, electrically conductive polymers can be tailored to delivery additional cues that the native tissues present. This approach will benefit the production of effective materials for a wide range of applications, such as regenerative medicine and long-term biocompatibility of the implants. In addition to electrical activity, important factors to be considered in fabricating biomimetic conductive biomaterials include mechanical rigidity, structures, and bioactivities. Note that biomimetic conductive materials should be designed and fabricated to achieve the best responses depending on the target biological system.

First, in comparison with the rigidity of conductive polymers $(>\mathrm{MPa})$, the most tissues are softness with a few $\mathrm{Pa}$ (brain tissues) to $\mathrm{kPa}$ except bone [19]. This mechanical mismatch not only causes poor direction of cellular responses but also inflammatory tissue reactions [20]. For example, cells and tissues, especially stem cells, generally exhibit the fast and specified growth and differentiation when cultured with the materials present similar mechanical properties to the native tissues [21]. Hence, this indicates the needs of developing softer and flexible conductive materials. To this end, composite hybrid materials were fabricated by mixing or growing conductive polymers with elastic materials or hydrogels. Interestingly, conductive hydrogels can mimic the mechanical softness of the native soft tissues by presenting tens of $\mathrm{kPa}$ of Young's modulus [22]. Yet, lowering the rigidity accompanies the impairment of electrical conductivities, which has to be overcome in the future studies. Still, since some biomedical applications need small currents, the conductive hydrogels will be useful.

Another important property is a structure, which can serve as biomimetic features of extracellular matrices including porosity, nano-/micro-structures, and orientation [23]. Advances in technologies enable the fabrication of such structurally effective conductive materials, which allow for the production of biologically important subcellular scaled features. For example, electron beam lithographic patterns of PPy could facilitate the polarization of embryonic hippocampal neurons [24]. Also, various fibrous structures of conductive polymers could be obtained by direct electrospinning, phase separation, and nano-coating of nanofibrous mats [25]. Interestingly, recent studies done by Hardy et al. demonstrated the enhanced osteogenesis of human mesenchymal stem cells cultured on PPy-silk nanofibers by electrical stimulation [26], suggesting the cooperative or additive roles of topographical cues and electrical cues as biomimetic functional materials. Likewise, a variety of conductive nanofibers have been produced for different types of cells.

Lastly, various biological active molecules are to be immobilized in/or conductive polymers by physical or chemical fashions. Since the effect interactions are often mediated by receptor-ligand binding, it will be critical to fabricate biologically active conductive materials by immobilizing ECM proteins, polysaccharides, and growth factors. In particular, anioinic proteins and mucopolysaccharides can be doped into oxidized conductive polymers, which can be easily produced during polymerization processes of conductive polymers [27]. Covalent immobilization of ECM proteins and growth factors enables prolonged interactions with cells without substantial consumption and signal regulation via binding with integrins and tyrosine kinase receptors, respectively. These signaling pathways finally affect gene expression levels of various genes related with proliferation, survival, and differentiation. For example, nerve growth factor immobilization onto conductive polymers have been attempted to enhance neural cell differentiation [28]. Interestingly, growth factors and electrical stimulation through conductive scaffolds could act together to induce the neurite formation and elongation. In addition, various ECM-derived peptides (e.g., Arg-Gly-Asp) were also incorporated into conductive polymer scaffolds to better mimic the native ECM, which could support attachment, growth and differentiation of various cells, such as neural cells [29], endothelial cells [30], cardiac cells [31], and fibroblasts [32]. For example, cell membrane mimicking conductive polymer having IKVAV (Ile-Lys-Val-Ala-Val) peptide and phosphorylcholine promoted neurite outgrowth and protein secretion on neural cells [33].

In summary, biomimetic conductive polymers can offer great promise to actively modulate biological systems. Still, better mimicry of characteristics of the target tissues and their demonstration will be desirable. High performance conductive polymer-based biomaterials will be greatly beneficial for applications of tissue engineering, drug delivery, and biocompatible bioelectrodes.

\section{Biomimetic medical materials trends: design of biomaterials for nerve tissue engineering}

The design of optimal biomaterials for regenerating injured human tissues and organs plays a crucial role in tissue engineering and regenerative medicine, especially for highly complex nerve system [33, 34]. Biomimetics, a science of mimicking natural phenomena of a biological 
system in terms of its composition, structure and function as a model for engineering new materials and systems, has been a promising strategy for inspiring the design and fabrication of novel biomaterials for tissue engineering and regenerative medicine. From the viewpoint of biomimetics, new generation of biomaterials should serve as not only structural support, but also artificial microenvironments that mimic the natural stem cell niches to deliver stem-cell-regulatory signals to purposefully stimulate specific cellular responses at the molecular level for mediating the regeneration of living tissues with full restoration of normal structure and function [33-35]. Biomaterial properties, such as the material architecture, mechanical properties, surface topographies, chemical properties, and biological ligands have been widely proven to exert influences as regulatory cues on stem-cell fate both in vivo and in vitro.

Previous studies indicate that biophysical properties of biomaterials are very important design parameters in preparing artificial regenerative niche for nerve regeneration. For example, the stiffness/elasticity of the underlying substrate could direct stem cell fate and even regulate their lineage differentiation [36, 37]. Adult neural stem cells exhibited promoted neuronal differentiation on soft substrates (100-500 Pa), while stiffer substrates $(1,000-10,000 \mathrm{~Pa})$ led to glial differentiation. Besides, it has been suggested that mesenchymal stem cells (MSCs) fate can also be directed by matrix elasticity of $0.1 \sim 1 \mathrm{kPa}$ toward neural differentiation. Therefore, the biomaterials designed for neural tissue regeneration should have lower stiffness to mimic the soft ECM of the neural tissue, which has positive effects on spontaneous neural differentiationof endogenous and/or exogenous stem cells. Furthermore, it has been widely accepted that aligned microstructures of biomaterials are of vital importance in nerve tissue engineering based on biomimetic ideas. Natural nerve tissues, not like most of other living tissues, have hierarchically oriented structures from a single neural axon to nerve fibers that are closely related to the directional transmission of nerve impulses. Previous studies have shown that the aligned structure was extensively identified in designing nerve conduits due to their ability of promoting alignment and elongation in regenerating axons, and regulating preferential neural differentiation of stem cells [38-40]. Beyond that, aligned fibrous structures provided a topographical cue to stimulate the elongation and enhanced neuronal differentiation of both adult neural stem cells [41] and MSCs [42] compared to random fibers. However, most of the previous studies applied 2-dimensional (2D) aligned fibrous membranes or meshes in cell culture that actually have a prominent difference on the stiffness of nerve tissues. Therefore, a suitable biomaterial for nerve regeneration should be soft with lower elasticity resembled natural neural matrix, and at the same time has 3-dimensional (3D) aligned structures.

In our study, a hierarchically aligned fibrillar fibrin hydrogel that was fabricated through electrospinning and the concurrent molecule self-assembly process mimics the soft and oriented features of nerve tissue simultaneously, thus providing hybrid biophysical cues to instruct cell behavior in vitro and in vivo. We found that the low elasticity and aligned topography of AFG had co-effects on promoting the neurogenic differentiation of human umbilical cord mesenchymal stem cells (hUMSCs), and also inducing dorsal root ganglions neurons to project numerous long neurite outgrowths longitudinally along the fibers rapidly for total migration distance of $1.96 \mathrm{~mm}$ in three days without the supplement of neurotrophic factors. Moreover, the aligned fibrin hydrogel implanted in a rat T9 dorsal hemisection spinal cord injury model was found to promote endogenous neural cells fast migration and axonal invasion along the fibers constructing aligned tissue cable in vivo. Our results suggest that matrix stiffness and aligned topography could synergistically instruct stem cell neurogenic differentiation and rapid neurite outgrowth, providing great promising in biomaterials design for applications in nerve regeneration.

\section{Competing interests}

The authors declare that they have no competing interests.

\section{Authors' contributions}

SMZ, KDP and ISN organized and worked on the 2016 Korea-China Joint Symposium on Biomimetic Medical Materials and organized the manuscript. The authors in each mini-reviews and communications contributed their corresponding parts: while KMP and KDP worked on polymeric hydrogel, JYP and SMW did design of electrical polymers for biomimetics and design of biomaterials for nerve tissue engineering, respectively. ISN has organized all the parts of this manuscript and all the authors read and approved the final manuscript.

Ki Dong Park, Ph.D., Ajou University, Korea

Ki Dong Park is a full professor of department of molecular science and technology, applied chemistry, and biomedical Engineering at Ajou University, Korea. Dr. Park earned his B.S. and M.S. degrees in the industrial chemistry, Hanyang University in 1981 and 1983. He received his Ph.D. degree in pharmaceutics, University of Utah in 1990. He pursued his postdoctoral training in pharmaceutics/center for controlled chemical delivery at University of Utah from 1990 to 1991. After his POSTDOC training, he worked as a principle research scientist at Korea Institute of Science and Technology (KIST) from 1991 to 2000. Finally, he joined Ajou University as an associate professor in 2000. His research is focusing on developing advanced polymeric biomaterials (e.g., injectable polymeric materials and blood/tissue compatible materials), which can be applied into a wide range of biomedical applications, including tissue engineering, polymeric drug carriers, artificial organs, and medical devices. Xiumei Wang, Ph.D., Tsinghua University, Beijing, China

Xiu-Mei Wang is a full professor at the institute for regenerative medicine and biomimetic materials of the school of materials science and engineering,

Tsinghua University, China. Dr. Wang received her B.S. and Ph.D degrees in materials science \& engineering in 2000 and 2005 from Tsinghua University. After her PhD, She worked as a postdoctoral fellow in the center for musculoskeletal research at University of Rochester (U.S.A.) from July 2005 to May 2006 and in the center for biomedical engineering at Massachusetts Institute of Technology (U.S.A.) from May 2006 to January 2008. She then joined Tsinghua University in 2008. Her current research focuses on tissue engineering 
and regenerative medicine, including nerve regeneration, bone regeneration, angiogenesis, and biomaterial-stem cell interactions.

Jae-Young Lee, Ph.D, Gwangju Institute of Science and Technology (GIST), Korea Jae-Young Lee is an assistant professor of the school of materials science and engineering, Gwangju Institute of Science and Technology (GIST), Korea. Dr. Lee received B.S. and M.S. degrees in the chemical technology, Seoul National University in 1997 and 1999. He worked as an associate research manager in LG Life Science Ltd. from 1999 to 2005. He received his Ph.D. from The University of Texas at Austin in 2010. He studied his postdoctoral research in University of California Berkeley with an American Heart Association (AHA) postdoctoral fellowship. He joined GIST in 2012. His research is in the field of biomaterials, which include biomimetic material design, electrically active biomaterials, plasmonic nanomaterials, and stem cell applications.

Kyung Min Park, Ph.D., Incheon National University, Korea

Kyung Min Park is an assistant professor of the division of bioengineering at the Incheon National University, Korea. Dr. Park received his B.S. and MS degrees in the applied chemistry and the biomedical engineering, respectively, Ajou University. He received his Ph.D. in the molecular science and technology (BK21) at the same university in 2012. After Ph.D., he pursued his post-doctoral training in the chemical and biomolecular engineering at the Johns Hopkins University from 2012 to 2015. Finally, he joined the Incheon National University in 2015. His current research focuses on designing novel hydrogel materials for a broad range of biomedical applications, including tissue regeneration, drug delivery as well as engineered healthy/disease models.

Shengmin Zhang, Ph.D., Huazhong University of Science and Technology, China Dr. Shengmin Zhang received his Ph.D. in Biomedical Materials from Wuhan University of Technology, China. He became the Chair Professor and Director of the Advanced Biomaterials and Tissue Engineering Center at Huazhong University of Science and Technology in Wuhan, China, from 2003. He became a professor at the Materials Science Department in Wuhan University of Technology from 2000. Prof. Zhang has over 20-year experience in biomaterials and tissue engineering fields and is a chairman of Regenerative Medical Materials Committee, Chinese Society for Biomaterials (CSBM) and CSBM Chairman of Science Popularization Committee. He serves on the editorial boards of several well-established international journals, such as Tissue Engineering (Part A, B and C, USA), Biomedical Materials (IOP, UK), etc. Insup Noh, Ph.D., Seoul National University of Science and Technology, Korea Insup Noh is a professor of the department of chemical and biomolecular engineering and a founder of convergence institute of biomedical engineering and biomaterials in Seoul National University of Science and Technology, co-operated with Korea Institute of Radiological and Medical Sciences, from 1999. He earned his MSE and Ph.D. degrees at University of Texas at Austin, USA, in 1995 and 1997, respectively. He worked in both California Institute of Technology as a special graduate student from 1995 to 1997 and in Division of Harvard-MIT Health Science and Technology as a postdoc from 1997 to 1999. He is currently vice presidents of both the Korean Society for Biomaterials and the Korean Tissue Engineering and Regenerative Medicine Society, serving as an editor-in-chief of Biomaterials Research as well as an editors advisory group of BioMed Central.

\section{Acknowledgement}

These works have been developed from the 2015 Korea-China Joint Symposium on Biomimetic Medical Materials, Seoul, Korea. This work was supported by the Grantsof both the National Research Foundation of Korea (NRF) (2014K2A2A7060928), Basic Science Research Program through the NRF grant funded by the Ministry of Science, ICT \& Future Planning (2015R1A2A1A14027221), Republic of, and the Natural Science Foundation of China (NSFC; No.81461148032).

\section{Author details}

${ }^{1}$ Department of Molecular Science and Technology, Ajou University, 206 Worldcup-ro, Yeongtong-gu, Suwon 16499, Korea. ${ }^{2}$ School of Materials Science and Engineering, Tsinghua University, Beijing 100084, China. ${ }^{3}$ School of Materials Science and Engineering, Gwangju Institute of Science and Technology (GIST), Gwangju 500-715, Korea. ${ }^{4}$ College of Life Science and Bioengineering, Incheon National University, Incheon 22012, Korea. ${ }^{5}$ Advanced Biomaterials and Tissue Engineering Center, Huazhong University of Science and Technology, Wuhan, P. R. China. ${ }^{6}$ Convergence Institute of Biomedical Engineering and Biomaterials, Seoul National University of Science and Technology, Seoul 01811, Korea. ${ }^{7}$ Department of Chemical and Biomolecular Engineering, Seoul National University of Science and Technology, 232 Gongneung-ro, Nowon-gu, Seoul 01811, Korea.
Received: 11 January 2016 Accepted: 24 February 2016 Published online: 29 March 2016

\section{References}

1. LV S, Dudek DM, Cao Y, et al. Designed biomaterials to mimic the mechanical properties of muscles. Nature. 2010;465:69-73.

2. He J, Wang XM, Spector M, Cui FZ. Scaffolds for central nervous system tissue engineering. Front Mater Sci. 2012;6:1-25.

3. Lutolf MP, Gilbert PM, Blau HM. Designing materials to direct stem-cell fate. Nature. 2009:462:433-41

4. Onoe H, Okitsu T, Itou A, Kato-Negishi M, Gojo R, Kiriya D, et al. Metre-long cell-laden microfibres exhibit tissue morphologies and functions. Nat Mater. 2013;12:584-90.

5. Guilak F, Cohen DM, Estes BT, Gimble JM, Liedtke W, Chen CS. Control of stem cell fate by physical interactions with the extracellular matrix. Cell Stem Cell. 2009;5:17-26.

6. Park KM, Gerecht S. Hypoxia-inducible hydrogels. Nat Commun. 2014;5:4075

7. Engler AJ, Sen S, Sweeney HL, Discher DE. Matrix elasticity directs stem cell lineage specification. Cell. 2006;126:677-89.

8. Hurtado A, Cregg JM, Wang HB, Wendell DF, Oudega M, Gilbert RJ, et al. Robust CNS regeneration after complete spinal cord transection using aligned poly-L-lactic acid microfibers. Biomaterials. 2011;32:6068-79.

9. Han S, Wang B, Jin W, Xiao Z, Li X, Ding W, et al. The linear-ordered collagen scaffold-BDNF complex significantly promotes functional recovery after completely transected spinal cord injury in canine. Biomaterials. 2015;41:89-96.

10. Jiang X, Cao HQ, Shi LY, Ng SY, Stanton LW, Chew SY. Nanofiber topography and sustained biochemical signaling enhance human mesenchymal stem cell neural commitment. Acta Biomaterialia. 2012;8:1290-302.

11. Lim SH, Liu XY, Song H, Yarema KJ, Mao HQ. The effect of nanofiber-guided cell alignment on the preferential differentiation of neural stem cells. Biomaterials. 2010;31:9031-9.

12. Cho YI, Choi JS, Jeong SY, Yoo HS. Nerve growth factor (NGF)-conjugated electrospun nanostructures with topographical cues for neuronal differentiation of mesenchymal stem cells. Acta Biomaterialia. 2010;6:4725-33.

13. Wallace GG, Higgins MJ, Moulton SE, Wang C. Nanobionics: the impact of nanotechnology on implantable medical bionic devices. Nanoscale. 2012;4:4327-47.

14. McCaig CD, Rajnicek AM, Song B, Zhao M. Has electrical growth cone guidance found its potential? Trends Neurosci. 2002;25(7):354-59.

15. Patel S, Kurpinski K, Quigley R, Gao H, Hsiao BS, Poo MM, et al. Bioactive nanofibers: synergistic effects of nanotopography and chemical signaling on cell guidance. Nano Lett. 2007;7:2122-28.

16. Pelto J, Bjorninen M, Palli A, Talvitie E, Hyttinen J, Mannerstrom B, et al. Novel polypyrrole-coated polylactide scaffolds enhance adipose stem cell proliferation and early osteogenic differentiation. Tissue Eng Pt A. 2013; 19(7-8):882-92.

17. Guimard NK, Gomez N, Schmidt CE. Conducting polymers in biomedical engineering. Prog Polym Sci. 2007;32(8-9):876-921.

18. Hardy JG, Lee JY, Schmidt CE. Biomimetic conducting polymer-based tissue scaffolds. Curr Opin Biotech. 2013;24:847-54.

19. Cox TR, Erler JT. Remodeling and homeostasis of the extracellular matrix: implications for fibrotic diseases and cancer. Dis Model Mech. 2011;4:165-78.

20. Green RA, Lovell NH, Wallace GG, Poole-Warren LA. Conducting polymers for neural interfaces: challenges in developing an effective long-term implant. Biomaterials. 2008;29:3393-99.

21. Engler AJ, Sen S, Sweeney HL, Discher DE. Matrix elasticity directs stem cell lineage specification. Cell. 2006;126:677-89.

22. Ding $H$, Zhong M, Kim YJ, Pholpabu P, Balasubramanian A, Hui CM, et al. Biologically derived soft conducting hydrogels using heparin-doped polymer networks. ACS Nano. 2014;8:4348-57.

23. Shekaran A, Garcia AJ. Nanoscale engineering of extracellular matrixmimetic bioadhesive surfaces and implants for tissue engineering. Biochim Biophys Acta. 2011;1810(3):350-60.

24. Gomez N, Lee JY, Nickels JD, Schmidt CE. Micropatterned polypyrrole: a combination of electrical and topographical characteristics for the stimulation of cells. Adv Funct Mater. 2007;17:1645-53.

25. Lee JY. Electrically conducting polymer-based nanofibrous scaffolds for tissue engineering applications. Polym Rev. 2013;53:443-59.

26. Hardy JG, Geissler SA, Aguilar D, Villancio-Wolter MK, Mouser DJ, Sukhavasi RC, et al. Instructive conductive 3D silk foam-based bone tissue scaffolds enable 
electrical stimulation of stem cells for enhanced osteogenic differentiation. Macromol Biosci. 2015;11:1490-6.

27. Ma Z, Mao Z, Gao C. Surface modification and property analysis of biomedical polymers used for tissue engineering. Colloid Surf B. 2007;60:137-57.

28. Lee JY, Bashur CA, Milroy CA, Forciniti L, Goldstein AS, Schmidt CE. Nerve growth factor-immobilized electrically conducting fibrous scaffolds for potential use in neural engineering applications. IEEE Trans Nanobiosci. 2012;11(1):15-21.

29. Povlich LK, Cho JC, Leach MK, Corey JM, Kim J, Martin DC. Synthesis, copolymerization and peptide-modification of carboxylic acid-functionalized 3,4-ethylenedioxythiophene (EDOTacid) for neural electrode interfaces. Biochim Biophys Acta. 2013;1830(9):4288-93.

30. Lee JW, Serna F, Nickels J, Schmidt CE. Carboxylic acid-functionalized conductive polypyrrole as a bioactive platform for cell adhesion. Biomacromolecules. 2006; 7(6):1692-5.

31. Kai D, Prabhakaran MP, Jin G, Ramakrishna S. Polypyrrole-contained electrospun conductive nanofibrous membranes for cardiac tissue engineering. J Biomed Mater Res A. 2011;99(3):376-85.

32. Shi G, Rouabhia M, Wang Z, Dao LH, Zhang Z. A novel electrically conductive and biodegradable composite made of polypyrrole nanoparticles and polylactide. Biomaterials. 2004;25(13):2477-88

33. Zhu B, Luo SC, Zhao H, Lin HA, Sekine J, Nakao A, et al. Large enhancement in neurite outgrowth on a cell membrane-mimicking conducting polymer. Nat Commun. 2014;5:4523.

34. Shinde UP, Yeon B, Jeong B. Recent progress of in situ formed gels for biomedical applications. Prog Polym Sci. 2013;38:672-701.

35. Seliktar D. Designing cell-compatible hydrogels for biomedical applications. Science. 2012;336:1124-8.

36. Park KM, Ko KS, Joung YK, Shin H, Park KD. In situ cross-linkable gelatin-poly (ethylene glycol)-tyramine hydrogel via enzyme-mediated reaction for tissue regenerative medicine. J Mater Chem. 2011;21:13180-7.

37. Nguyen MK, Alsberg E. Bioactive factor delivery strategies from engineered polymer hydrogels for therapeutic medicine. Prog Polym Sci. 2014;39:1236-65.

38. Cushing MC, Anseth KS. Hydrogel cell cultures. Science. 2007:316:1133-4.

39. Huebsch N, Mooney DJ. Inspiration and application in the evolution of biomaterials. Nature. 2009:462:426-32.

40. Place ES, Evans ND, Stevens MM. Complexity in biomaterials for tissue engineering. Nat Mater. 2009;8:457-70.

41. Highley CB, Rodell CB, Burdick JA. Direct 3D printing of shear-thinning hydrogels into self-healing hydrogels. Adv Mater. 2015;27:5075-9.

42. Murphy SV, Atala A. 3D bioprinting of tissues and organs. Nat Biotech. 2014; 32:773-85.

\section{Submit your next manuscript to BioMed Central and we will help you at every step:}

- We accept pre-submission inquiries

- Our selector tool helps you to find the most relevant journal

- We provide round the clock customer support

- Convenient online submission

- Thorough peer review

- Inclusion in PubMed and all major indexing services

- Maximum visibility for your research

Submit your manuscript at www.biomedcentral.com/submit

C Biomed Central 